\title{
NONLINEAR CONTROLLER DESIGN OF A SHIP AUTOPILOT
}

\author{
MiROSŁAW TOMERA \\ Department of Ship Automation \\ Gdynia Maritime University, ul. Morska 83, 81-225 Gdynia, Poland \\ e-mail: tomera@am.gdynia.pl
}

\begin{abstract}
The main goal here is to design a proper and efficient controller for a ship autopilot based on the sliding mode control method. A hydrodynamic numerical model of CyberShip II including wave effects is applied to simulate the ship autopilot system by using time domain analysis. To compare the results similar research was conducted with the PD controller, which was adapted to the autopilot system. The differences in simulation results between two controllers are analyzed by a cost function composed of a heading angle error and rudder deflection either in calm water or in waves. Simulation results show the effectiveness of the method in the presence of nonlinearities and disturbances, and high performance of the proposed controller.
\end{abstract}

Keywords: sliding mode control, nonlinear control, disturbance rejection, ship control.

\section{Introduction}

When a ship sails on the sea, its autopilot system is usually used for leading the ship along the desired course via automatic changes in the rudder blade deflection. In recent years, numerous publications have been devoted to investigating those properties of autopilots which would make it possible to steer the ship both in calm water and in the presence of additional disturbances, such as wind, sea waves, and/or sea currents. The motion of the ship is highly susceptible to the action of the approaching waves and, consequently, the ship, which always sails in the presence of waves, will behave in a different way from that defined for calm water. The ship behaviour in various environmental conditions is predicted using mathematical models developed to model kinetic movements of the ship and the dynamics of the phenomena taking place (Fang and Luo, 2005).

The ship motion is described by a set of six complicated differential equations which describe six degrees of freedom. The models used for designing control systems change depending on the goals of the control. These goals can be divided, in a most general way, into low-speed positioning and high-speed steering. The first type is named dynamic positioning (DP) and includes keeping constant position and low-speed manoeuvering. For a DP systems, the model with six degrees of freedom is reduced to a simpler one with three degrees of freedom. This model is li- near in the kinetic part. High-speed steering includes automatic steering along the desired course, steering along the trajectory (high speed position tracking) and path following. In those applications, centripetal and Coriolis forces, along with the nonlinear viscous effect, are dominating, and that is why the kinetic model is nonlinear. For ships moving at a constant speed only the first approximation of viscous damping is taken into account, and therefore in this case linear approximations of ship dynamics can be used (Fossen, 2002). A historical overview of the development of mathematical models of ships was given by Clark (2003).

Nowadays we can observe great interest in mathematical models of ships, in particular those developed in the time domain, as they can be used in designing control systems and computer simulations. Models of this type can be found in (Galbas, 1988; Gierusz, 2001; Fossen, 1994; Kallstrom and Ottosson, 1982; Skjetne et al., 2004).

A conventional autopilot system used for controlling the ship motion is a PD controller with constant parameters values. These controllers can work properly in precisely defined operating conditions, but the quality of their work is worse when these conditions change. Ship dynamic characteristics can change as a consequence of changes of the ship speed, load, and external disturbances such as waves, wind, and/or sea currents. In many cases manual tuning of control parameters is necessary. Therefore a lot of research activities have been oriented to improving 


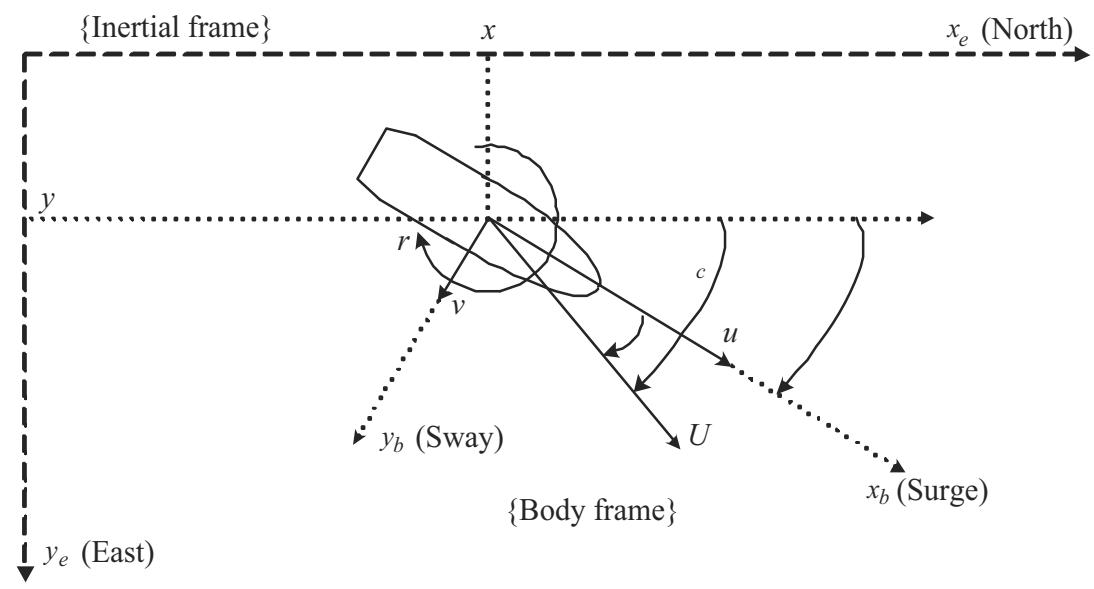

Fig. 1. Inertial earth-fixed frame and the body-fixed frame for a ship with the earth-fixed position $(x, y)$, the heading $\psi$, the ship course $\psi_{c}$, the sideslip $\beta$, the corresponding body-fixed velocities $(u, v)$, and the rotation rate $r$ (Skjetne et al., 2004).

the quality of operation of these controllers using adaptive mechanisms which automatically change ship model parameters, depending on operating conditions. Ship model parameters are estimated directly and then used for tuning the controller's parameters (Fang and Luo, 2005).

Automatic control systems of the ship motion often make use of sliding mode controllers, which are known for their good quality of control. This type of controller is rather well documented and bases on switching control, which delivers an additional steering action when plant dynamics characteristics change due to nonlinearities (Castillo-Toledo et al., 2008; Etien et al., 2002; Fossen, 1994; Healey and Lienard, 1993; Healey and Marco, 1992; McGookin et al., 2000; Sadati and Ghadami, 2008; Slotine and Li, 1991; Solea and Nunes, 2007; Tomera and Smierzchalski, 2006). The structure of the sliding mode controller includes a nominal part and a nonlinear switching part. Switching makes the controller highly resistant to direct external and internal changes due to the environment.

Various implementation methodologies for sliding mode controllers exist. Like other conventional control structures, the design of sliding-mode controllers needs the knowledge of the mathematical model of the plant, which decreases the performance in some applications where mathematical modeling of the system is very difficult and where the system has a wide range of parameter variations together with unexpected and sudden external disturbances. For those cases we need so-called "intelligent" controllers. The idea of combining these intelligent control structures with the sliding-mode approach has attracted many researchers. These controllers mainly work based on neural networks (Bagheri and Moghaddam, 2009; Hung and Chung, 2007), or fuzzy control (Bessa et al., 2008; Chen et al., 2009). Also new methods based on genetic algorithms are available (Demir- tas, 2009; Moghaddam and Bagheri, 2010).

In the present research, the sliding mode controller was adapted to analysing properties of the ship autopilot in the situation when the ship is steered in waves. The analysis was made using the physical model of a ship called CyberShip II, which provides services to drilling platforms (Lindegaard and Fossen, 2002; Sveen, 2003; Skjetne et al., 2004). In order to compare the obtained results, reference tests were performed using the PD controller. Characteristics of the examined controllers were tested in calm water and in the presence of sea waves with course changes generated by the change in turning points on the trajectory of the moving ship. The cost function took into account the ship course error and rudder blade deflection, and was used for evaluating the quality of the steering action of the controllers.

\section{Mathematical model of ship dynamics}

The motion of the ship is described using nonlinear differential equations in six degrees of freedom (DOFs). The variables $(x, y, z)$ are respectively referred to as surge, sway and heave, describing the position in the threedimensional space, and the variables $(\phi, \theta, \psi)$, called roll, pitch and yaw, are used for describing the orientation of the ship. Assuming that the ship is stable in surge and sway directions, with small amplitudes $\phi=\theta=\dot{\phi}=\dot{\theta} \approx$ 0 we can neglect the dynamics of roll and pitch. Likewise, for a ship sailing on water surfaces with $z \approx 0$ on the average we can also neglect the heave dynamics. The resulting model describing the ship motion in the horizontal plane becomes a model with three degrees of freedom. The ship motion is described in the inertial frame fixed to the Earth's coordinate system, called NED (North-EastDown), and another coordinate frame attached to the moving ship (Fig. 1). 
The state variables describing the ship motion are collected in two vectors (Fossen, 1994), $\eta=[x, y, \psi]^{\mathrm{T}}$ and $\nu=[u, v, r]^{\mathrm{T}}$, where $(x, y)$ denotes the position coordinates, $\psi$ is the heading (yaw) angle, $(u, v)$ are the bodyfixed linear velocities (surge and sway) of the ship, and $r$ is the yaw rate of the ship. The Earth-fixed velocity vector defined in the inertial frame is related to the body-fixed velocity vector through the following kinematic relationship:

$$
\dot{\eta}=R(\psi) \nu
$$

where $R(\psi)$ is the rotation matrix, calculated from the formula

$$
R(\psi)=\left[\begin{array}{ccc}
\cos (\psi) & -\sin (\psi) & 0 \\
\sin (\psi) & \cos (\psi) & 0 \\
0 & 0 & 1
\end{array}\right]
$$

The ship model selected for designing and testing the control system with the sliding mode controller was the mathematical model of the ship CyberShip II, developed at the Department of Engineering Cybernetics, Norwegian University of Science and Technology (NTNU), Trondheim, Norway. The physical model of this ship sails in the Marine Cybernetics Laboratory (MCLab), NTNU, (http: / / www.itk.ntnu.no/marinkyb/MCLab).

2.1. Mathematical model of CyberShip II. The physical model called Cybership II is a scale replica of a supply ship, made at a scale of 1:70. Its mass is $m=23.8$ $\mathrm{kg}$, the overall length is $L_{O A}=1.255 \mathrm{~m}$, and the breadth is $B=0.29 \mathrm{~m}$. This model was equipped with two main propellers and two rudders aft, and one bow thruster. The propellers and the thruster are controlled by the rotational speed. In a general form, the mathematical model of this ship is given by the formula

$$
M \dot{\nu}+C(\nu) \nu+D(\nu) \nu=\tau \text {. }
$$

The system inertia matrix $M=M_{R B}+M_{A}$ includes the rigid-body system inertia matrix $M_{R B}$, and the hydrodynamic matrix included added mass coefficients $M_{A}$. The coefficients of the matrix $M_{R B}$ were calculated using the data obtained from direct measurements and basic ship characteristics, such as dimensions, weight, mass distribution, volume, etc. The coefficients in the matrix $M_{A}$ are connected with the water surface effect and are evaluated from semi-empirical formulas (Faltinsen, 1990):

$$
M=\left[\begin{array}{ccc}
m-X_{\dot{u}} & 0 & 0 \\
0 & m-Y_{\dot{v}} & m x_{g}-Y_{\dot{r}} \\
0 & m x_{g}-N_{\dot{v}} & I_{z}-N_{\dot{r}}
\end{array}\right]
$$

The Coriolis-centripetal matrix $C(\nu)$ includes Coriolis and centripetal terms $C_{R B}(\nu)$ acting on the ship treated as a solid body, as well as hydrodynamic Coriolis and centripetal terms $C_{A}(\nu)$ connected with the fluid in which the ship moves (Fossen, 2002):

$$
C(\nu)=C_{R B}(\nu)+C_{A}(\nu),
$$

where

$$
\begin{aligned}
C_{R B}(\nu) & =\left[\begin{array}{ccc}
0 & 0 & -m\left(x_{g} r+v\right) \\
0 & 0 & m u \\
m\left(x_{g} r+v\right) & -m u & 0
\end{array}\right], \\
C_{A}(\nu) & =\left[\begin{array}{ccc}
0 & 0 & c_{13}(\nu) \\
0 & 0 & -c_{23}(\nu) \\
-c_{13}(\nu) & c_{23}(\nu) & 0
\end{array}\right], \\
c_{13}(\nu) & =Y_{\dot{v}} v+\frac{1}{2}\left(N_{\dot{v}}+Y_{\dot{r}}\right) r \text { and } c_{23}(\nu)=X_{\dot{u}} u .
\end{aligned}
$$

The damping matrix $D(\nu)$ is connected with the hydrodynamic damping forces. It consists of the linear part $D_{L}$, determined for a selected small and constant surge velocity $\nu=\nu_{0} \approx\left[u_{0}, 0,0\right]^{\mathrm{T}}$ (Fossen, 2002), and of the nonlinear part $D_{N L}(\nu)$, which makes it possible to determine hydrodynamic damping forces at high velocities (Skjetne, et al., 2004):

$$
D(\nu)=D_{L}+D_{N L}(\nu),
$$

where

$$
\begin{aligned}
& D_{L}=\left[\begin{array}{ccc}
-X_{u} & 0 & 0 \\
0 & -Y_{v} & -Y_{r} \\
0 & -N_{v} & -N_{r}
\end{array}\right] \\
& D_{N L}(\nu)=\left[\begin{array}{ccc}
-d_{11}(\nu) & 0 & 0 \\
0 & -d_{22}(\nu) & -d_{23}(\nu) \\
0 & -d_{32}(\nu) & -d_{33}(\nu)
\end{array}\right] \\
& d_{11}(\nu)=X_{|u| u}|u|+X_{u u u} u^{2} \\
& d_{22}(\nu)=Y_{|v| v \mid}|v|+Y_{|r| v}|r| \\
& d_{23}(\nu)=Y_{|v| r \mid}|v|+Y_{|r| r \mid}|r| \\
& d_{32}(\nu)=N_{|v| v}|v|+Y_{|r| v|r|} \\
& d_{33}(\nu)=N_{|v| r}|v|+N_{|r| r \mid}|r|
\end{aligned}
$$

The vector of forces acting on the ship's hull refers to the forces $\tau_{t h}$ generated by the propellers and rudders installed on the physical model of CyberShip II and to the forces $\tau_{w}$ generated by acting disturbances:

$$
\tau=\left[\tau_{X}, \tau_{Y}, \tau_{N}\right]^{\mathrm{T}}=\tau_{t h}+\tau w .
$$

\subsection{Mathematical models of propellers and rudder} blades. For rotating propellers with a fixed blade rudder deflection angle, the generated thrust force is more or less proportional to the square of the rotational speed $\omega_{i}$ of the shaft. For small speeds, the propeller/blade model can be 
divided into two parts, of which the first one describes the nominal thrust (blade deflection angles $\delta_{i}=0, i=1,2$ ):

$$
T_{i}=\left\{\begin{aligned}
k_{i T p} \omega_{i}^{2} & \text { for } \quad \omega_{i} \geqslant 0, \\
k_{i T n}\left|\omega_{i}\right| \omega_{i} & \text { for } \quad \omega_{i}<0,
\end{aligned}\right.
$$

$i=\{1,2,3\}$. The second part refers to additional lift and drag forces, generated by rudder blades connected with the propeller screws, $i=1,2$,

$$
\begin{gathered}
L_{i}=\left\{\begin{array}{cc}
T_{i}\left(1+k_{i L n} \omega_{i}\right)\left(k_{i L \delta 1}+k_{i L \delta 2}\left|\delta_{i}\right|\right) \delta_{i}, & \omega_{i} \geqslant 0, \\
0, & \omega_{i}<0,
\end{array}\right. \\
D_{i}=\left\{\begin{array}{cc}
T_{i}\left(1+k_{i D n} \omega_{i}\right)\left(k_{i D \delta 1}\left|\delta_{i}\right|+k_{i D \delta 2} \delta_{i}^{2}\right), & \omega_{i} \geqslant 0, \\
0, & \omega_{i}<0 .
\end{array}\right.
\end{gathered}
$$

For the above-mentioned three rotating propellers and two rudders, the following surge and sway forces are obtained:

$$
u=\left[\begin{array}{c}
T_{1}\left(\omega_{1}, \delta_{1}\right)-D_{1}\left(\omega_{1}, \delta_{1}\right) \\
T_{2}\left(\omega_{2}, \delta_{2}\right)-D_{2}\left(\omega_{2}, \delta_{2}\right) \\
T_{3}\left(\omega_{3}\right) \\
L_{1}\left(\omega_{1}, \delta_{1}\right) \\
L_{2}\left(\omega_{2}, \delta_{2}\right)
\end{array}\right] .
$$

Now we can write the vector of forces applied to the hull depending on the distribution of the propellers and rudder blades:

$$
\tau_{t h}=T u,
$$

where the matrix $T$ is the actuator configuration matrix:

$$
T=\left[\begin{array}{ccccc}
0 & 0 & 0 & 0 & 0 \\
0 & 0 & 0 & 0 & 0 \\
\left|L_{y T 1}\right| & -\left|L_{y T 2}\right| & \left|L_{x T 3}\right| & -\left|L_{x R 1}\right| & -\left|L_{x R 2}\right|
\end{array}\right]
$$

Numerical values of the parameters used in the presented mathematical model of CyberShip II can be found in (Lindegaard, 2003; Sveen, 2003; Skjetne et al., 2004; Skjetne, 2005).

2.3. Environmental disturbances. Three main types of environmental disturbances can be named, including

(i) waves generated by the wind,

(ii) ocean currents,

(iii) the wind.

The present analysis limits the list of examined disturbances to surface waves generated by the wind, i.e., the disturbances which are most important for the motion of a surface ship. The model which was used for simulating the movement of the ship in waves introduces forces and moments induced by regular sea on a ship having the shape of a block. It creates a vector $\tau_{w}=\left[X_{w}, Y_{w}, N_{w}\right]^{T}$, which is directly added to the input vector $\tau$ using the superposition principle (Fossen, 1994):

$$
\begin{aligned}
& X_{w}(t)=\sum_{i=1}^{N} \rho g B L T \cos (\beta) s_{i}(t), \\
& Y_{w}(t)=\sum_{i=1}^{N} \rho g B L T \sin (\beta) s_{i}(t), \\
& N_{w}(t)=\sum_{i=1}^{N} \frac{1}{24} \rho g B L\left(L^{2}-B^{2}\right) \sin (2 \beta) s_{i}^{2}(t),
\end{aligned}
$$

where $L$ and $B$ are the ship length and breath, respectively, $T$ is the average draught of a ship treated as blockshaped, $\rho$ is water density, $s_{i}(t)$ is the wave slope, while $\beta=\psi_{w}-\psi$ is the angle between the direction of the acting waves $\psi_{w}$ and the ship heading $\psi$, (in radians). The wave slope $s_{i}$ can be related to the wave spectral density function $S\left(\omega_{i}\right)$.

Various spectral densities can be used to calculate $S(\omega)$. Here, the modified Pierson-Mostkowitz spectrum was used (Fossen, 1994):

$$
S(\omega)=\frac{4 \pi^{3} H_{s}^{2}}{\left(0.710 T_{o}\right)^{4} \omega^{5}} \exp \left(\frac{-16 \pi^{3}}{\left(0.710 T_{o}\right)^{4} \omega^{4}}\right),
$$

where $T_{o}$ is the modal period $\left(T_{o}=0.80 \mathrm{~s}\right), H_{s}$ is the significant wave height $\left(H_{s}=5 \mathrm{~mm}\right)$. The simulated waves had the significant wave height equal to $3 \mathrm{~m}$, which corresponds to sea conditions 5 (rough sea).

\section{Control system for ship course tracking}

The reported investigations concerned the control system for a ship steering on its course. The input signal in the examined control system was the desired course $\psi_{d}$, which resulted from the covered trajectory segment. In a real coordinate system it can be calculated from the following relation:

$$
\psi_{d}=\operatorname{arctg}\left(\frac{y_{k+1}-y_{k}}{x_{k+1}-x_{k}}\right),
$$

where $\left(x_{k}, y_{k}\right)$ are the coordinates of the way point passed by the ship, while $\left(x_{k+1}, y_{k+1}\right)$ are the coordinates of the next way point. The task of the controller is to generate the control signal $\delta_{z}$ for the steering gear. In the examined ship course control system two controllers were taken into account, i.e., the PD controller and the SMC (sliding mode control) controller.

To evaluate the quality of operation of the examined controllers, the cost function was defined as

$$
\begin{aligned}
J & =\psi_{E}+\delta_{E}=\sum_{i=0}^{N}\left(\Delta \psi_{i}\right)^{2}+\lambda \sum_{i=0}^{N} \delta_{i}^{2} \\
& =\sum_{i=0}^{N}\left[\left(\Delta \psi_{i}\right)^{2}+\lambda \delta_{i}^{2}\right] .
\end{aligned}
$$


The first term of this function refers to the course change, where $N$ is the number of iterations during the simulation process, $\lambda$ is the weight coefficient, $\Delta \psi_{i}$ is the $i$-th course error calculated as the difference between the desired course and the ship heading, $\delta_{i}$ is the $i$-th deflection of the rudder blade. The main task of the weight coefficient $\lambda$ is to gain the course error term at the same level as the term representing the rudder deflection. Without external disturbances, an decreasing course error will increase the rudder blade deflection and vice versa: a decreased rudder blade deflection will increase the course error.

3.1. PD controller. The controller which, due to its simplicity, is most frequently used in autopilot systems is the PD controller. It controls the rudder blade deflection depending on the values of the heading error and the yaw rate. The PD controller is described by the following formula:

$$
\delta_{z}=K_{P}\left(\psi-\psi_{d}\right)+K_{D} r
$$

where $K_{P}$ and $K_{D}$ are controller settings, $\psi_{d}$ and $\psi$ are the desired and current ship headings, respectively, $r=\mathrm{d} \psi / \mathrm{d} t$ is the yaw rate of the ship, and $\delta_{z}$ is the commanded rudder blade deflection.

3.2. Sliding mode controller. Sliding control belongs to the group of robust control methods, as changes in the dynamics of the controlled object and external disturbances do not lead to noticeable deterioration of the control quality. The control action performed using the sliding mode controller consists in tracking the desired trajectory, which is obtained by comparing the current values $x$ of the controlled states with the desired values $x_{z}$, i.e., by calculating the tracking error $e=x_{z}-x$. The structure of the sliding mode controller consists of the equivalent part and an additional part used for compensating the action of disturbances. The equivalent part is usually a linear controller designed for the linearized plant and responsible for the main control action. The additional part is a switching controller, in which the minimization of the sliding surface value takes place as a function of the tracking error $\sigma_{h}(e)$. The control consists in reducing the control error to zero, $e=0$, which is equivalent to the reduction of the sliding surface value to zero. The output signal of the sliding mode controller, being the input signal for the controlled object, is composed of two terms,

$$
\delta_{z}=u_{e q}+u_{s w},
$$

where $u_{e q}$ is the signal of the equivalent part and $u_{s w}$ is the signal of the switching part.

3.2.1. Equivalent part. For the purposes of the linear controller synthesis, being part of the examined sliding mode controller, simplified mathematical models of CyberShip II were worked out. From a ship moving with a constant surge speed $u=u_{0}$, the above nonlinear model of CyberShip II (3) can be simplified. The surge dynamics are separated from the above mathematical model assuming the port/starboard symmetry. The steering forces are usually linearly dependent on the rudder blade deflection $\delta$, according to the formulas $\tau_{Y}=-Y_{\delta} \delta$ and $\tau_{N}=-N_{\delta} \delta$.

As a result, the manoeuvring model is obtained which consists of the surge dynamics

$$
\begin{aligned}
\left(m-X_{\dot{u}}\right) \dot{u} & -X_{u}\left(u-u_{0}\right)-\left(m-X_{\dot{v}}\right) v r \\
& -\left(m x_{g}-\frac{1}{2} N_{\dot{v}}-\frac{1}{2} Y_{\dot{r}}\right) r^{2}=\tau_{X}
\end{aligned}
$$

and the yaw-sway dynamics

$$
M \dot{\nu}+N\left(u_{0}\right) \nu=b \delta,
$$

where $\nu=[v, r]^{\mathrm{T}}$ is the state vector, $\delta$ is the rudder angle and

$$
\begin{aligned}
M & =\left[\begin{array}{cc}
m-Y_{\dot{v}} & m x_{g}-Y_{\dot{r}} \\
m x_{g}-N_{\dot{v}} & I_{z}-N_{\dot{r}}
\end{array}\right], \\
N\left(u_{0}\right) & =\left[\begin{array}{cc}
-Y_{v} & -Y_{r}+\left(m-X_{\dot{u}}\right) u_{0} \\
-N_{v} & -N_{r}+\left(m x_{g}-\frac{1}{2} N_{\dot{v}}-\frac{1}{2} N_{\dot{v}}\right) u_{0}
\end{array}\right], \\
b & =\left[\begin{array}{c}
Y_{\delta} \\
N_{\delta}
\end{array}\right] .
\end{aligned}
$$

The matrix $N\left(u_{0}\right)$ is obtained by the summation of linear damping $D_{L}$ and Coriolis and centripetal terms $C\left(u_{0}\right)$, that is,

$$
N\left(u_{0}\right)=C\left(u_{0}\right)+D_{L}
$$

For each fixed surge speed $u=u_{0}$, the above characteristics remain linear. That is why $u$ is considered a parameter. Equation (23) constitutes a linearly parameterized model, written in the form of the Davidson and Schiff (1946) model. This model can then be converted to the Nomoto models described by Clarke (2003).

To eliminate the sway speed $v$ from Eqn. (23) two other alternative descriptions of the Davidson and Schiff model are proposed by Nomoto et al. (1957). This way we arrive at the Nomoto transfer function, which represents the relation between the ship course $\psi$ and the rudder blade deflection $\delta$ :

$$
\frac{\psi(s)}{\delta(s)}=\frac{K\left(1+T_{3} s\right)}{s\left(1+T_{1} s\right)\left(1+T_{2} s\right)} .
$$

The parameters of the transfer function are related to the hydrodynamic coefficients according to the following 
relations:

$$
\begin{aligned}
T_{1} T_{2}= & |M| /|N|, \\
T_{1}+T_{2}= & \left(n_{11} m_{22}+n_{22} m_{11}\right. \\
& \left.-n_{12} m_{21}-n_{21} m_{12}\right) /|N|, \\
K_{R}= & \left(n_{21} b_{1}-n_{11} b_{2} /|N|,\right. \\
K_{R} T_{3}= & \left(m_{21} b_{1}-m_{11} b_{2} /|N|,\right. \\
K= & -K_{R},
\end{aligned}
$$

where the coefficients $m_{i j}, n_{i j}$ and $b_{i}(i=1,2 ; j=1,2)$ are the components of the matrices $M, N$ and $B$ in (22), $|M|$, $|N|$ are determinants of the matrices $M$ and $N$, respectively.

The Nomoto model can be reduced by determining the substitute time constant using the relation $T=T_{1}+$ $T_{2}-T_{3}$ :

$$
\frac{\psi(s)}{\delta(s)}=\frac{K}{s(1+T s)},
$$

and it can be written in the form of the following state equations:

$$
\begin{gathered}
{\left[\begin{array}{c}
\dot{\psi} \\
\dot{r}
\end{array}\right]=\left[\begin{array}{cc}
0 & 1 \\
0 & a_{22}
\end{array}\right]\left[\begin{array}{l}
\psi \\
r
\end{array}\right]+\left[\begin{array}{c}
0 \\
b_{2}
\end{array}\right] \delta,} \\
\psi=\left[\begin{array}{ll}
1 & 0
\end{array}\right]\left[\begin{array}{l}
\psi \\
r
\end{array}\right]+[0] \delta,
\end{gathered}
$$

where $r=\mathrm{d} \psi / \mathrm{d} t, a_{11}=-1 / T, b_{2}=K / T$.

The synthesis of the sliding mode controller was done based on the simplified Nomoto model (33), (34) written in the form of the following dynamic equations:

$$
\begin{gathered}
\dot{x}_{h}=A_{h} x_{h}+B_{h} \delta, \\
\psi=C_{h} x_{h}+D_{h} \delta .
\end{gathered}
$$

The sliding surface $\sigma_{h}$ was defined as

$$
\sigma_{h}=h_{1}\left(\psi_{z}-\psi\right)+h_{2}\left(r_{z}-r\right),
$$

where $h_{1}$ and $h_{2}$ are the elements of the right eigenvector $h, r_{z}$ is the commanded yaw rate, and $\psi_{z}$ is the commanded ship heading. In order to stabilize the ship on its course, a two-element vector $K=\left[k_{1}, k_{2}\right]^{\mathrm{T}}$ was selected in such a way that

$$
A_{c}=A_{h}-B_{h} K^{\mathrm{T}}=\left[\begin{array}{cc}
0 & 1 \\
-b_{2} k_{1} & a_{22}-b_{2} k_{2}
\end{array}\right] \text {. }
$$

Two eigenvalues of the closed system provide opportunities for determining the required gains $k_{1}$ and $k_{2}$. After calculating the matrix $A_{c}$, the required eigenvector $h$ is calculated as the solution of the equation $A_{c}^{\mathrm{T}} h=0$ for the eigenvalue of the smaller absolute value (module).
In the article, the equivalent part of the sliding mode controller was executed using the linear controller whose action was based on the following formula:

$$
u_{e q}=-N \psi_{z}+K x,
$$

where $N$ is the gain to scale the commanded heading $\psi_{z}$, which is calculated from

$$
\begin{gathered}
N=N_{u}+K N_{x}, \\
{\left[\begin{array}{c}
N_{x} \\
N_{u}
\end{array}\right]=\left[\begin{array}{ll}
A_{h} & B_{h} \\
C_{h} & D_{h}
\end{array}\right]^{-1}\left[\begin{array}{l}
0 \\
1
\end{array}\right] .}
\end{gathered}
$$

3.2.2. Switching part. The switching part of sliding mode control takes the form

$$
u_{s w}=h^{\mathrm{T}} \dot{x}_{h z}-\eta_{h} \operatorname{tgh}\left(\frac{\sigma_{h}}{\phi_{h}}\right),
$$

where $\dot{x}_{h z}=\left[r_{z}, \dot{r}_{z}\right]$ is the required state vector, in which $\dot{r}_{z}=\mathrm{d} r_{z} / \mathrm{d} t$ is the desired yaw acceleration, $\eta_{h}$ is the switching gain and $\phi_{h}$ is the boundary layer thickness.

3.2.3. Complete form. Finally, the control law of the sliding mode controller is calculated from the following formula:

$$
\begin{aligned}
\delta_{z}= & u_{e q}+u_{s w} \\
= & -N \psi_{z}+K x \\
& +\left[h^{\mathrm{T}} \dot{x}_{h z}-\eta_{h} \tanh \left(\frac{\sigma_{h}}{\phi_{h}}\right)\right] .
\end{aligned}
$$

The commanded ship heading $\psi_{z}$ and the commanded yaw rate $r_{z}$ were determined using the reference model described by

$$
\begin{aligned}
{\left[\begin{array}{c}
\dot{\psi}_{z} \\
\dot{r}_{z}
\end{array}\right]=} & {\left[\begin{array}{cc}
0 & 1 \\
-\omega_{n}^{2} & -2 \zeta \omega_{n}
\end{array}\right]\left[\begin{array}{l}
\psi_{z} \\
r_{z}
\end{array}\right] } \\
& +\left[\begin{array}{c}
0 \\
\omega_{n}^{2}
\end{array}\right] \psi_{d},
\end{aligned}
$$

where $\psi_{d}$ is the desired ship course resulting from the assumed trajectory segment.

In (37) we defined the switching surface for the sliding mode controller that is based on the state variable errors. The control objective is to drive the system state to the commanded state. The feedback gain vector $K$ in the equivalent part was computed by means of pole placement by first specifying the closed-loop matrix $A_{c}$. In the design of the sliding mode controller, an equivalent control is first given so that the states can stay on the sliding surface.

The sliding controller is divided into an equivalent control input and a reaching mode control input if the switching form is negative (Fossen, 1994). 
Comparing Eqns. (20) and (43), we can see the difference between these two controllers. The commanded rudder blade deflection, determined in the PD controller, is only calculated from the heading error and the yaw rate. In the sliding mode controller, the commanded rudder blade deflection is calculated from the course error, the yaw rate, the commanded yaw rate, and the commanded yaw acceleration.

\section{Simulation tests}

The simulation tests of the course control systems making use of the here discussed controllers were carried out for two cases: in calm waters and in the presence of waves corresponding to the sea conditions equal to 5 on the Beaufort scale. The model selected for the test was the mathematical model of CyberShip II. The rotational speed of the propeller screws was constant and equal to $\omega_{1}=\omega_{2}=$ $8.13 \mathrm{rev} / \mathrm{s}$. The ship moved at a constant surge speed equal to $0.3 \mathrm{~m} / \mathrm{s}$ in calm waters, at zero rudder blade deflections. For simplicity, the water depth was assumed infinite.

The problem which was to be solved concerned tuning the parameters of the PD controller and the sliding mode controller. For the PD controller, two parameters were to be selected: the course error gain $K_{P}$ and the ship's yaw rate gain $K_{D}$. At the same time, for the sliding mode controller, four parameters were to be selected, i.e., two eigenvalues $\left(\lambda_{1}, \lambda_{2}\right)$ of the closed system, the switching gain $\eta_{h}$ and the boundary layer thickness $\phi_{h}$. The eigenvalues $\left(\lambda_{1}, \lambda_{2}\right)$ of the closed system make it possible to calculate the gain vector $K$ and the nominal eigenvector $h$. These calculations were performed using the linearized Dawidson and Schiff model (22). As a result, for CyberShip II, moving with a constant surge speed $u=u_{0}=$ $0.3 \mathrm{~m} / \mathrm{s}$, the following linearized mathematical model was obtained (and used for further calculations):

$$
\begin{aligned}
& {\left[\begin{array}{rr}
33.8000 & 1.0948 \\
1.0948 & 2.7600
\end{array}\right]\left[\begin{array}{l}
\dot{v} \\
\dot{r}
\end{array}\right]} \\
& +\left[\begin{array}{rr}
0.8897 & 14.9900 \\
2.3687 & 2.9948
\end{array}\right]\left[\begin{array}{l}
v \\
r
\end{array}\right]=\left[\begin{array}{r}
0.4304 \\
-0.2363
\end{array}\right] \delta .
\end{aligned}
$$

The model described by (45) was transformed to the Nomoto model, described by the dynamic equations (33), (34), which in the examined case took the form

$$
\begin{aligned}
{\left[\begin{array}{c}
\dot{\psi} \\
\dot{r}
\end{array}\right] } & =\left[\begin{array}{ll}
0 & 1.0000 \\
0 & 0.1057
\end{array}\right]\left[\begin{array}{l}
\psi \\
r
\end{array}\right]+\left[\begin{array}{r}
0.0000 \\
-0.0040
\end{array}\right] \delta, \\
\psi & =\left[\begin{array}{ll}
1 & 0
\end{array}\right]\left[\begin{array}{l}
\psi \\
r
\end{array}\right]+[0] \delta .
\end{aligned}
$$

The obtained linearized mathematical model of CyberShip II (46), (47) was used for calculating gains in the linear controller using the pole location method. The obtained eigenvalues of the system closed by the matrix of gains from state coupling were equal to $\lambda_{1}=-0.0005$, $\lambda_{2}=-7.8128$. The formula (43) includes the eigenvector $h$ corresponding to the eigenvalue $\lambda_{1}$. For the nonlinear part of the sliding mode controller (43), the following values were assumed: $\eta_{h}=10, \phi_{h}=0.3$, while for the PD controller (20), the assumed gains were $K_{P}=2$ and $K_{D}=50$. The parameters in the reference model (44) were $\omega_{n}=0.1, \zeta=0.85$.

Table 1. Cost function value (in calm water).

\begin{tabular}{|l|c|c|c|}
\hline Algorithm & $\psi_{E}$ & $\delta_{E}$ & $J$ \\
\hline \hline PD controller & 128.20 & 128.74 & 256.94 \\
\hline SMC controller & 117.07 & 117.42 & 234.49 \\
\hline
\end{tabular}

The examined control systems were modeled in Matlab/Simulink, and the simulations were carried out in time domain. The numerical integration was performed using the fourth-order Runge-Kutta method with the integration step equal to $0.1 \mathrm{~s}$. The object used for simulations was the nonlinear mathematical model of CyberShip II, described in detail in (Lindegaard and Fossen, 2002; Sveen, 2003; Skjetne et al., 2004).

The simulated waves had a significant height equal to $3 \mathrm{~m}$, which corresponds to the sea conditions equal to 5 on the Beaufort scale. The waves were modelled based on the modified Pierson-Mostkowitz spectrum (Fossen, 1994). For the purpose of wave simulation for CyberShip II, whose physical model was made at a scale of $1: 70$, the modal period was assumed, after scaling, as being equal to $\left(T_{o}=0.80 \mathrm{~s}\right)$, while the significant height of the wave was equal to $H_{s}=5 \mathrm{~mm}$, the average draught of CyberShip II is equal to $T=0.05 \mathrm{~m}$. The direction of the wave action assumed for the calculations was $\psi_{w}=180^{\circ}$.

The systems were first tested in calm water. The results of the simulations are shown in Fig. 2. The left-hand column collects the data recorded for the control system with the PD controller, while in right-hand column-for the control system with a sliding mode controller. The upper diagrams show the time-histories of changes in surge speeds, the central diagrams-heading changes, and the lower diagrams - real rudder blade deflections.

The presented figures reveal how different formulas used for calculating control signals affect the behaviour of the entire control system. At a sudden change of the commanded heading, the PD controller, described by (20), immediately calculates a high value of the commanded signal, which then leads to fast deflection of the rudder blade and a resultant decrease in the surge velocity of the ship. At the same time, in the sliding mode controller, the use of the reference model makes the steering signal change more slowly. As a consequence, the rudder blade does not deflect so rapidly and the decrease in the surge veloci- 

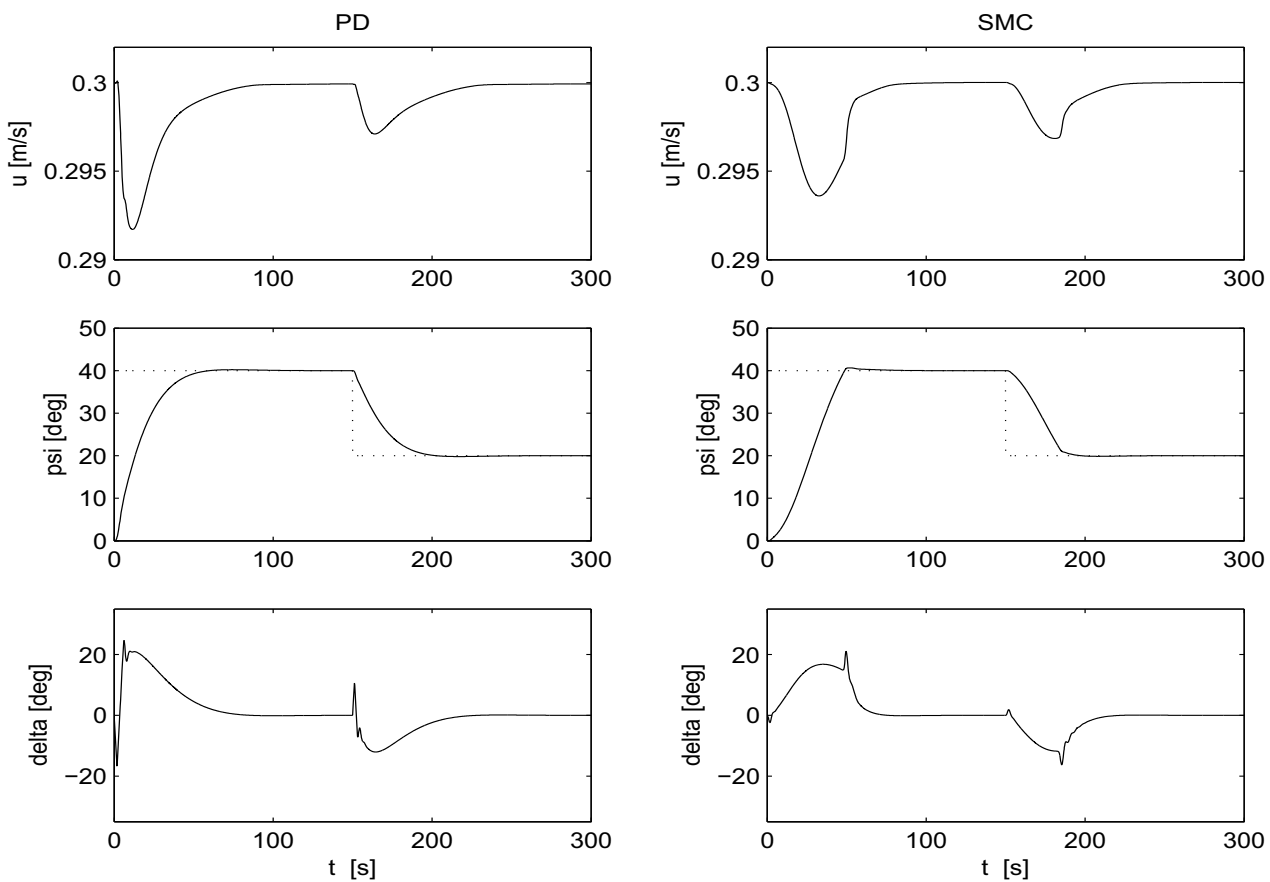

Fig. 2. Motion response for simulation in calm waters: left—PD controller, right-SMC controller.
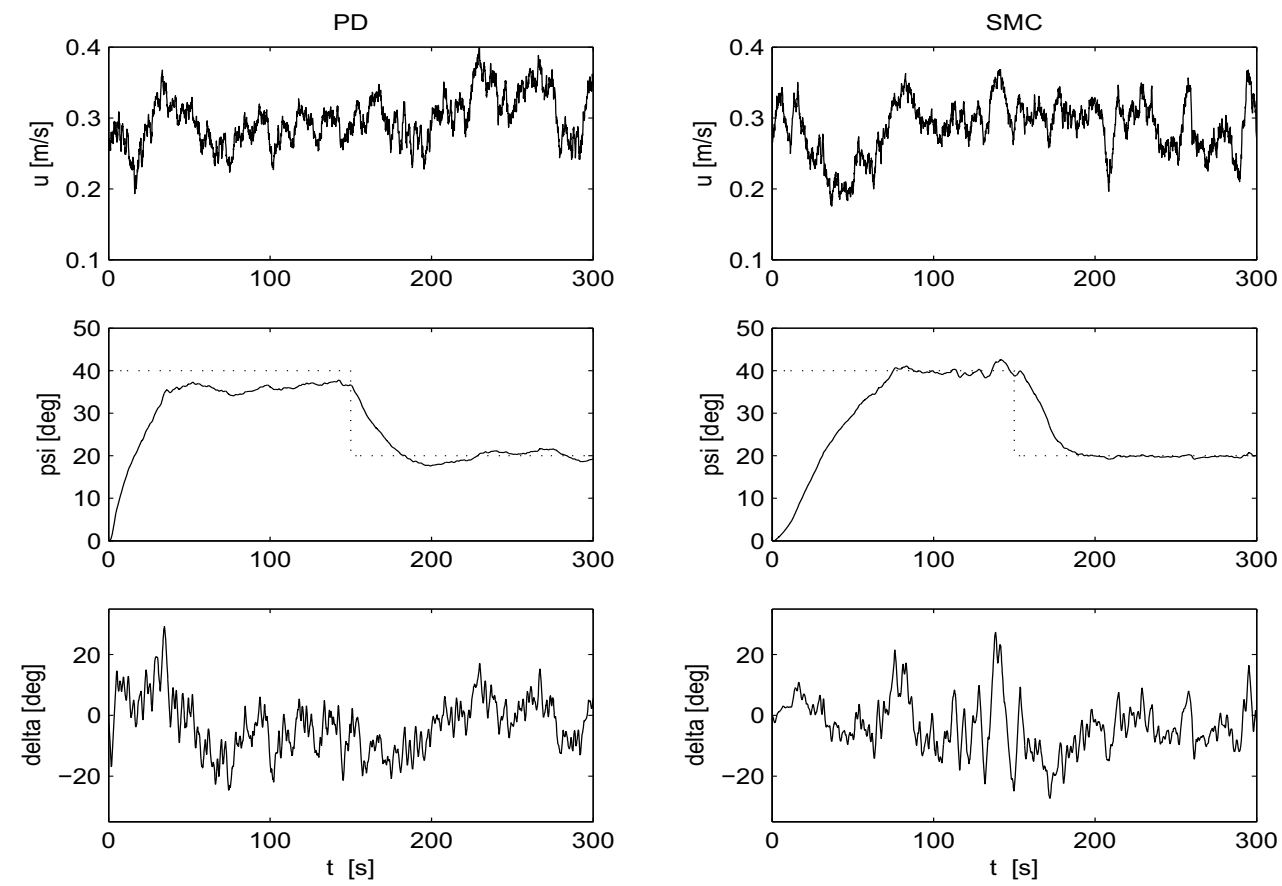

Fig. 3. Motion response for simulation in rough sea: left-PD controller, right-SMC controller.

ty of the ship is smaller.

The values of the determined steering cost function are collected in Table 1. It is noticeable that all performance indices determined for the sliding mode controller are smaller than those calculated for the PD controller.

Figure 3 shows the results of simulations on a regu- lar 3-meter high wave, for unchanged parameters of both controllers. The obtained results differ remarkably from those recorded in calm water.

Comparing the course changes presented in the central diagrams, we can see that the presence of the switching element, having the integrating function in the sli- 
Table 2. Cost function value (in regular waves).

\begin{tabular}{|l|c|c|c|}
\hline Algorithm & $\psi_{E}$ & $\delta_{E}$ & $J$ \\
\hline \hline PD controller & 259.38 & 261.40 & 520.78 \\
\hline SMC controller & 194.57 & 195.98 & 390.55 \\
\hline
\end{tabular}

ding mode controller, makes it possible to track the ship along the desired course much more effectively than in the case of the PD controller, which does not have the integrating function.

The values of steering performance indices determined for the case of ship steering in the presence of a regular wave are collected in Table 2. Comparing them with those in Table 1, we can notice that it is much more difficult to track the ship in the presence of waves than in calm water, because the cost function values for steering on waves are much higher than in the case of calm water.

\section{Conclusion}

The object of the investigations reported in the article was the course tracking ability by a ship sailing rough sea. Two controllers were examined, namely, the PD controller and the sliding mode controller, for which correct values of parameters were selected. All tests were performed at a constant rotational speed of the propeller screws situated at the stern. This speed was equal to $8.13 \mathrm{rev} / \mathrm{s}$. As a result, the ship moved with a constant surge speed equal to $0.3 \mathrm{~m} / \mathrm{s}$ in calm water, at zero rudder blade deflection. The performed computer simulations made it possible to conclude that both controllers properly led the ship in calm water, and similar cost function values were obtained.

The wave effect on ship autopilot operation is significant, which can be observed by comparing the timehistories shown in Figs. 2 and 3, and the cost function values of steering performance collected in Tables 1 and 2 . The investigations revealed that the sliding mode controller better tracks the heading at the presence of waves than the PD controller. The advantage of the use of switching in the sliding algorithm consists in the fact that is helps to keep precisely the current course by compensating disturbances generated by an external environment, such as the wave disturbances analyzed in the paper.

\section{References}

Bagheri, A. and Moghaddam, J.J. (2009). Simulation and tracking control based on neural-network strategy and slidingmode control for underwater remotely operated vehicle, Neurocomputing 72(7-9): 1934-1950.

Bessa, W.M., Dutra, M.S. and Kreuzer, E. (2008). Depth control of remotely operated underwater vehicles using an adaptive fuzzy sliding mode controller, Robotics and Autonomous Systems 56(8): 670-677.
Castillo-Toledo, B., Di Gennaro, S., Loukianov, A.G. and Rivera, J. (2008). Discrete time sliding mode control with application to induction motors, Automatica 44(12): 3036-3045.

Chen, C.-Y. Li, T.-H.S. and Yeh Y.-C. (2009). EP-based kinematic control and adaptive fuzzy sliding-mode dynamic control for wheeled mobile robots, Information Sciences 179 (1-2): 180-195.

Clarke, D. (2003). The foundations of steering and maneouvering, Proceedings of the IFAC Conference on Manoeuvering and Control Marine Crafts, Girona, Spain, pp. 10-25.

Davidson, K.S.M. and Schiff, L.I. (1946). Turning and course keeping qualities, Transactions-Society of Naval Architects Marine Engineers 54: 152-200.

Demirtas, M. (2009). DSP-based sliding mode speed control of induction motor using neuro-genetic structure, Expert Systems with Applications 36(3): 5533-5540.

Etien, E., Cauet, S., Rambault, L. and Champenois, G. (2002). Control of an induction motor using sliding mode linearization, International Journal of Applied Mathematics and Computer Science 12(4): 523-532.

Faltinsen, O.M. (1990), Sea Loads on Ships and Offshore Structures, Cambridge University Press, Cambridge.

Fang, M.-C. and Luo, J.-H. (2005). The nonlinear hydrodynamic model for simulating a ship steering in waves with autopilot system, Ocean Engineering 32(11-12): 1486-1502.

Fossen, T.I. (1994). Guidance and Control of Ocean Vehicles, John Wiley \& Sons Ltd., Chichester.

Fossen, T.I. (2002). Marine Control Systems: Guidance, Navigation, and Control of Ships, Rigs and Underwater Vehicles, Marine Cybernetics, Trondheim.

Galbas, J. (1988). Synthesis of precise ship steering systems making use of thrusters, Ph.D. thesis, Technical University of Gdańsk, Gdańsk, (in Polish).

Gierusz, W. (2001). Simulation model of the shiphandling training boat Blue Lady, Proceedings of Control Applications in Marine Systems, Glasgow, UK.

Healey, A.J. and Marco, D.B. (1992). Slow speed flight control of autonomous underwater vehicles: Experimental results with NPS AUV II, Proceedings of the 2nd International Offshore and Polar Conference, San Francisco, CA, USA, pp. 523-532.

Healey, A.J. and Lienard, D. (1993). Multivariable sliding mode control for autonomous diving and steering of unamanned underwater vehicles, IEEE Journal of Oceanic Engineering 18(3): 327-339

Hung, L. and Chung, H. (2007). Decoupled control using neural network-based sliding-mode controller for nonlinear systems, Expert Systems with Applications 32(4): 1168-1182.

Kallstrom, C. G. and Ottosson, P. (1982). The generation and control of roll motion of ships in close turns, Proceedings of the 4th International Symposium on Ship Operation and Automation, Genova, Italy, pp. 25-36.

Lindegaard, K.-P. and Fossen T.I. (2002). Fuel efficient rudder and propeller control allocation for marine craft: Experiments with model ship, IEEE Transactions on Control Systems Technology 11(6): 850-862. 
Lindegaard K.-P. (2003). Acceleration Feedback in Dynamic Positioning, Ph.D. thesis, Norwegian University of Science and Technology, Trondheim.

McGookin, E.W., Murray-Smith, D.J., Li, Y. and Fossen T.I. (2000). Ship steering control system optimisation using genetic algorithms, Control Engineering Practice 8(4): 429-443.

Moghaddam, J.J. and Bagheri, A. (2010). An adaptive neurofuzzy sliding mode based genetic algorithm control system for under water remotely operated vehicle, Expert Systems with Applications 37(1): 647-660.

Nomoto, K., Taguchi, T., Honda, K. and Hirano, S. (1957). On the steering qualities of ships, International Shipbuilding Progress 4(35): 354-370.

Sadati, N. and Ghadami, R. (2008). Adaptive multi-model sliding mode control of robotic manipulators using soft computing, Neurocomputing 71(13-15): 2702-2710.

Skjetne, R., Smogeli, O. and Fossen T.I. (2004). Modeling, identification, and adaptive maneuvering of Cybership II: A complete design with experiments, Proceedings of the IFAC Conference on Application Marine Systems, CAMS 2004, Ancona, Italy, pp. 203-208.
Skjetne, R. (2005). The Maneuvering Problem, Ph.D. thesis, Norwegian University of Science and Technology, Trondheim.

Slotine, J.J.E. and Li, W. (1991). Applied Nonlinear Control, Prentice Hall, London.

Solea, R. and Nunes, U. (2007). Trajectory planning and slidingmode control based trajectory-tracking for cybercars, Integrated Computer-Aided Engineering 14(1): 33-47.

Sveen, D.A. (2003). Robust and adaptive tracking control for synchronization with an ROV: Practical implementation on CyberShip II, M.Sc. thesis, Norwegian University of Science and Technology, Trondheim.

Tomera, M. and Śmierzchalski, R. (2006). Sliding controller for ship course steering, Proceedings of the 7th IFAC Conference on Manouvering and Control of Marine Craft, MCMC, Lisbon, Portugal.

Mirosław Tomera received the M.Sc. degree in automatic control in 1990 and the Ph.D. degree in ship automation in 2001 from the Gdańsk University of Technology. Since 1990 he has been working at Gdynia Maritime Academy, where he lectures on control theory. His research interests include the design of autopilots for ships, dynamic positioning systems, state estimators for marine vessels and trajectory tracking control. He is an author and co-author of about 60 papers. 